\title{
¿Tres son multitud? Algunas notas sobre el Third Party Funding y su aplicación en arbitrajes comerciales ${ }^{(* *)}$
}

\author{
Three is a crowd? Some notes about Third Party Funding and its \\ application in commercial arbitrations
}

\author{
EL PACTO PARTE DE UNA PREMISA SENCILLA: SI LA POSICIÓN DEL LITIGANTE ES AMPARADA, \\ ENTONCES EL FONDO FINANCISTA RECIBE UN PORCENTAJE O FRACCIÓN DE LO OBTENIDO \\ (O RECUPERADO) POR EL LITIGANTE QUE GANÓ EL CASO. A DIFERENCIA DE UN PRÉSTAMO \\ ORDINARIO, EL DEMANDANTE QUE ES FINANCIADO CON ESTE MECANISMO NO TIENE QUE \\ PAGARLE AL PRESTAMISTA SI PIERDE EL CASO O NO LOGRA RECUPERAR SUMA ALGUNA.
}

"When the money is coming your way, you don't ask any questions" Frank Underwood ${ }^{(1)}$

Resumen: En este artículo, el autor nos introduce a la institución del third party funding y su futura aplicación en los litigios arbitrales. El third party funding permite que los costos del litigante puedan ser financiados. Asimismo, señala los problemas que presenta el tercero financista, y la implementación de normas para afrontar esa situación. Finalmente, hay una tendencia de ampliar el deber de revelación de las partes sobre si se está siendo financiado por un tercero.

Palabras clave: Third Party Funding - Arbitraje - Costos del litigio Financiamiento - Litigación - Arbitraje Internacional

Abstract: In this article, the author introduces us to the institution of third party funding and its future application in arbitration litigation. Third party funding allows the litigant's costs can be funded. Moreover, he points out the problems that the third party financing has, and the implementation of

$\left({ }^{*}\right) \quad$ Abogado por la Pontificia Universidad Católica del Perú. Asociado de Bullard, Falla, Ezcurra + Abogados. Adjunto de docencia en la Pontificia Universidad Católica del Perú y en la Universidad del Pacífico. Miembro Extraordinario de la Asociación IUS ET VERITAS. Correo electrónico: crios@bullardabogados.pe

${ }^{* *}$ Nota del Editor: este artículo fue recibido el 2 de junio y su publicación fue aprobada el 25 de junio del mismo año. 


\section{¿Tres son multitud? Algunas notas sobre el Third Party Funding y su aplicación en arbitrajes comerciales Three is a crowd? Some notes about Third Party Funding and its application in commercial arbitrations}

rules to deal with this situation. Finally, there is a tendency to expand the duty of disclosure of the parties whether a third party is funding them.

Keywords: Third Party Funding - Arbitration - Litigation Costs Funding - Litigation - International Arbitration

\section{Introducción}

No es ningún secreto: litigar cuesta. A las incontables horas de tensión deberá sumarle los costos administrativos que le exigirán el Poder Judicial o los árbitros, según su elección. Añada los costos de producir u obtener los documentos y la evidencia necesaria para su caso, e incluya la factura de costosos peritos o expertos técnicos. Y si la cuenta ya le parece grande, espere a que llegue la liquidación de honorarios de los abogados que lo patrocinan.

Litigar cuesta. Y cuesta tanto que, en ciertos casos, no resulta posible iniciar una acción judicial o arbitral incluso cuando se tiene una posición favorable o un caso ganador. Los costos pueden llegar a ser prohibitivos.

Afortunadamente, la tendencia de pagar por nuestros propios litigios está cambiando. Están apareciendo agentes en el mercado dispuestos a financiar nuestra controversia, incluso asumiendo el riesgo de no obtener retorno. Aplicando la máxima a mayor riesgo, mayor ganancia, estos agentes financieros ofrecerán una oportunidad para que diversos operadores puedan presentar un reclamo sin tener que preocuparse por el costo de la aventura judicial o arbitral.

A este tipo de financiamiento se le denomina third party funding, y viene siendo utilizado desde hace ya un tiempo tanto en arbitrajes internacionales ${ }^{(2)}$ como en controversias judiciales foráneas. El concepto es relativamente simple: otro paga por mí el costo de litigar, a cambio de un porcentaje de lo ganado o recuperado. A primera vista, parece una figura que presenta muchos beneficios y ningún problema.

No obstante, esta figura no está exenta de polémica. Así, saltan inmediatamente algunas dudas como: ¿el financista también forma parte del proceso? ¿Puede la sentencia o laudo declarar un derecho en favor del financista? ¿Debo declarar que estoy siendo financiado por un tercero? ¿Y qué ocurre cuando se presentan conflictos de intereses?

Es importante entonces clarificar la aplicación y problemas que presenta esta figura, pues viene siendo empleada cada vez con mayor frecuencia a nivel internacional ${ }^{(3)}$ y sin duda será utilizada también en sede local, cambiando ciertos paradigmas sobre los litigios judiciales y arbitrales.

Por ello, en el presente artículo trataremos en detalle esta nueva alternativa y abordaremos aquellas interrogantes que pudieran surgir al aplicar esta figura en litigios arbitrales.

\section{2. ¿En qué consiste el third party funding?}

El third party funding es un acuerdo en donde un litigante recurre a un financiamiento por

(1) Fincher, David (director). House of Cards, 2014.

(2) Esta figura nace en Australia, siendo usada de forma predominante en dicho país y en el Reino Unido. Otros países europeos, Canadá y Estados Unidos también han venido implementando esta práctica, principalmente para litigios domésticos. En Australia, se estima que el uso de esta modalidad de financiamiento se presenta en el $14 \%$ de las demandas judiciales destinadas a una reparación económica, especialmente en las denominadas class actions. Para mayor detalle véase 'Improving the Envioronment for Business in Australia: A proposal for Reforming Oversight of Third Party Litigation Financing". Chamber Institute for Legal Reform, 2013. Se puede acceder a la versión digital a través del siguiente hipervínculo: http:// www.instituteforlegalreform.com/uploads/sites/1/Australia_TPLF_Final.pdf

(3) Cassandra Burke Robertson, "The impact of Third Party Funding on Transnational Litigation," Case W. Reserve Journal of International Law, vol. 44, (2011): 162; Bernardo Cremades "Third Party Funding: Investing in Arbitration," ICC Institute of World Business Law 4, vol. 8 (2011): 2. 


\section{Carlos Ríos Pizarro}

parte de una entidad que es ajena a su representación legal. Este agente externo, un third party funder, financia la representación legal para, posteriormente, obtener una ganancia o beneficio. El financista puede ser un banco, un fondo de inversión, una compañía de seguros o incluso un individuo particular ${ }^{(4)(5)}$.

El pacto parte de una premisa sencilla: si la posición del litigante es amparada, entonces el fondo financista recibe un porcentaje o fracción de lo obtenido (o recuperado) por el litigante que ganó el caso(6). A diferencia de un préstamo ordinario, el demandante que es financiado con este mecanismo no tiene que pagarle al prestamista si pierde el caso o no logra recuperar suma alguna ${ }^{(7)}$.

Sin embargo, y como bien advierte el profesor Fernández Masiá:

"Ni mucho menos todas las solicitudes de financiación son aceptadas. A este respecto, se ha señalado que únicamente entre un 5 y un $10 \%$ de los potenciales casos son financiados finalmente. La perfección de un contrato de financiación supone un estudio profundo de las pretensiones de la parte financiada, que prueba que la reclamación está bien fundamentada y como mínimo no puede tacharse de frívola. Las entidades financiadoras son empresas con una estructura compleja y que tratan de financiar únicamente los casos tras un análisis minucioso de los méritos de las reclamaciones" (Fernández 2016).
En algunos casos, dependiendo de la estructura del acuerdo de financiamiento, el financista puede llegar a tomar control o influenciar en aspectos de la representación legal, llegando incluso, aunque de forma muy excepcional, a tomar completo control del caso(8). Un ejemplo claro de ello son los clásicos reclamos que se presentan en Estados Unidos por responsabilidad extracontractual o protección al consumidor, donde los afectados suelen dejar la representación legal del caso en manos de un fondo de inversión pues consideran que éste está en mejor capacidad de obtener un mejor resultado económico.

Aplicar este sistema permite financiar un litigio incluso en la totalidad de los costos. Sin embargo, éste no es el único beneficio que presenta esta figura. Explica la profesora Lacroix que hay cuatro razones principales que impulsan la industria del third party funding alrededor del mundo. La primera, y más evidente, consiste en permitir a los individuos poder presentar reclamos que de otra forma no podrían costear. Ello facilita y por ende incrementa el acceso a la justicia de aquellos con menos recursos aunque, como bien indica Lacroix, el financista no suele tener fines tan altruistas ${ }^{(9)}$.

(4) Tripp Chapmany Daniel Kalderimis. Third party funding in international arbitration. Lessons from litigation? En Kluwer Arbitration. Publicado el 15 de diciembre de 2014. Sobre el particular, véase también: Paul Rubinen: "Third-party financing of litigation," Northern Kentucky Law Review, vol. 38:4, 674.

(5) Véase Victoria Shanon Sahani, "Judging Third Party Funding," UCLA Law Review 63 (2016): 391.

(6) Véase Victoria Shanon Sahani, Judging Third Party Funding.

(7) Es importante advertir que esta no es la única forma en la que se estructura un financiamiento bajo la modalidad de third party funding. La asunción total de costos y riesgos es, sin duda, la más representativa, más no la única. El pacto puede ser tan diverso en la distribución del riesgo como diversos los agentes financistas.

(8) Como indican Nieuwveld y Shanon, "algunos acuerdos de financiamiento de terceros son estructurados de tal modo que el financista se convierte en el demandante y la parte original del caso se desvincula del mismo. Es importante anotar, sin embargo, que los financistas no pueden 'comprar' un reclamo derivado de un tratado de inversión debido a los requisitos de jurisdicción y de fondo que contemplan este tipo de casos, como por ejemplo la nacionalidad del inversionista. Sobre el particular, véase Lisa Nieuwveld y Victoria Shanon, "Third Party Funding in International Arbitration," Kluwer Law International (2012): 8 y siguientes.

(9) Véase Kevin Lacroix. What's happening now? Litigation Funding Apparently. D\&O Diary, 2013. "Litigation funding proponents contend that the funding arrangements helps to level the playing field by allowing litigants to pursue lawsuits against better financed opponents, or simply allowing litigants to keep litigation costs off their balance sheet. It seems clear that as the 


\author{
¿Tres son multitud? Algunas notas sobre el Third Party Funding y su aplicación en arbitrajes \\ comerciales \\ Three is a crowd? Some notes about Third Party Funding and its application in commercial \\ arbitrations
}

En segundo lugar, muchas compañías insolventes y pequeñas compañías buscan financiamiento de terceros como una manera de poder afrontar el costo de un reclamo con posibilidades reales de éxito(10).Tercero, compañías grandes que son permanentemente demandadas (por ejemplo, compañías de seguro) buscan alternativas para que dichos litigios no afecten sus balances contables, y los financistas ofrecen ello por un precio fijo. $Y$ finalmente, "el riesgo financiero de los últimos años ha llevado a muchos inversionistas a buscar invertir en instrumentos que no dependan del mercado financiero, el precio de las acciones o la valoración de compañías. Sienten que, medidos los riesgos, tienen una inversión más segura en el largo plazo" (Shanon 2016).

En suma, la figura presenta múltiples beneficios. Sin embargo, un amplio sector de litigantes, académicos y árbitros han presentado no pocas objeciones a la aplicación y uso de la figura. Se ha señalado por ejemplo (aunque en nuestra opinión con poco asidero en la realidad) que este mecanismo de financiación puede provocar un aumento de la litigiosidad en el ámbito del arbitraje internacional|(11).

Otras críticas apuntan a dilemas éticos. Así, por una parte porque puede incidir de manera clara sobre las relaciones entre el cliente y su abogado, de tal manera que puede condicionar la toma de decisiones por parte del titular de la acción durante el arbitraje o incluso violar las normas de confidencialidad presentes en la relación del cliente y su representación. Por otro lado, se argumenta que la presencia de un tercero financiador puede suponer un conflicto de intereses respecto a los árbitros, lo que puede poner en entredicho de manera justificada su necesaria imparcialidad e independencia.
En nuestra opinión, los problemas denunciados se originan, básicamente, por la ausencia de una regulación clara y expresa sobre el financiamiento de terceros. Trataremos dicho asunto a continuación.

\section{Acerca de la (des) regulación del Third Party Funding en el arbitraje}

Los Third Party Funders son participantes indirectos en un arbitraje y, pese a ello, los participantes directos (jueces, árbitros e incluso la parte opositora y sus abogados) pueden desconocer plenamente del involucramiento de un tercero financista.

Pudiera parecer que, como cualquier otro participante indirecto (piénsese en un perito por ejemplo), el Third Party Funder debería estar sujeto a las mismas reglas del arbitraje que se encuentra financiando. Sin embargo, las apariencias engañan. Aplicarles estas reglas a los funders es más complejo de lo que parece.

$Y$ es que, si se analiza con detalle, el tercero financista casi nunca encaja dentro de los típicos roles de un proceso judicial o arbitral. No es, intencionalmente, parte del arbitraje o proceso $^{(12)}$ (para evitar efectos sobre ellos), tampoco es representante legal y mucho

litigation funding field grows, the funding companies are offering new approaches - for example, the defense side option that the Gerchen Keller firm will be offering, or the 'defense cost cover' that provided protection for prospective RBS claimants sufficient for them to be able to take on litigation in U.K. notwithstanding the 'loser pays' litigation model that prevails there".

(10) Véase Kevin Lacroix. What's happening now? Litigation Funding Apparently. D\&O Diary, 2013. .

(11) En este sentido, Guillerme defiende que la financiación por terceros aumenta el volumen de reclamaciones infundadas al mismo tiempo que incrementa el riesgo de una prolongación innecesaria de los procedimientos. Al respecto, véase Catherine Kessedjian, "Retour d'experience des juristes d'enterpirese," Le financiament de contentiuex par un tiers (Phanteon-Assas 2012), 32-33.

(12) La doctrina tradicional define parte como "aquel que pide en propio nombre (o en cuyo nombre se pide) la actuación de una voluntad de la ley, y aquel frente al cual es pedida. La idea de parte viene dada, por consiguiente, por la litis misma, por la relación procesal, por la demanda; no hay que irse a buscarla fuera de la litis y, en particular, a la relación sustantiva que es objeto de la controversia. Véase Giuseppe Chiovenda. Instituciones de Derecho Procesal Civil, vol. I, 284. Similar posición tiene el profesor Lino Palacio, quien precisa que "partes son quienes de hecho intervienen o figuran en el proceso 


\section{Carlos Ríos Pizarro}

menos testigo. No es amicus curiae y ciertamente no es juez, árbitro o una institución arbitral. No se ve beneficiado por el contrato discutido en un arbitraje (y por ende no tendrá el derecho de iniciar un reclamo o ser imputable) y no recibe ninguna cesión de derechos o créditos respecto del contrato discutido en arbitraje. Los funders son, estrictamente hablando, inversionistas, y ello los convierte en una figura totalmente atípica para la mayor parte de procedimientos judiciales y arbitrajes regulados en el mundo.

Lo que es peor, diversos académicos y juristas reconocen que rara vez las legislaciones arbitrales o judiciales regulan el financiamiento de terceros y fijan reglas para su uso(13). Los funders están sujetos, en buena cuenta, a las reglas (no siempre claras)de la buena fe procesal o a principios, más no a reglas de conducta específicas con consecuencias claras y determinadas.

Entonces, ¿qué reglas aplicamos para los third party funders y cuál es el límite de su actuación? Buscando responder estas interrogantes, en los últimos años se han dado algunos pasos importantes para generar reglas concretas que permitan a las partes y árbitros conocer la forma en la que deben actuar respecto al financiamiento de terceros.

Concretamente, en el año 2014 se modificaron las Directrices de la IBA para incorporar y precisar dos reglas que permitan atender los problemas relacionados a terceros financistas, conforme al siguiente detalle:

“(6) Relaciones

(b) Si una de las partes fuere una persona jurídica, cualquier persona jurídica o física que tenga una relación de control sobre dicha persona jurídica, o que tenga un interés económico directo en, o deba indemnizar a una parte por, el laudo que se vaya a emitir en el arbitraje, podrá considerarse que ostenta la identidad de dicha parte.

(7) El deber de las partes y del Árbitro

(a) Cada parte deberá informar al árbitro, al Tribunal Arbitral, a las demás partes y a la institución arbitral o a cualquier otra institución nominadora (si la hubiere) sobre cualquier relación directa o indirecta que hubiere entre el árbitro y la parte (o cualquier otra sociedad del mismo grupo de sociedades o un individuo con una relación de control sobre la parte en el arbitraje) o entre el árbitro y cualquier persona o entidad con un interés económico directo en, o un deber de indemnizar a la parte por, el laudo que se emita en el arbitraje. Cada parte informará a iniciativa propia lo antes posible" (Directrices IBA sobre Conflictos de Intereses en Arbitraje Internacional 2014).

En comentario a estas modificaciones, se explicó que:

"(...) Los terceros financiadores y las aseguradoras en relación a la disputa pueden tener un interés económico directo en el laudo, y por tanto pueden considerarse como equivalentes a la parte. A estos efectos, los términos 'tercero financiador' y 'aseguradora' se refieren a cualquier persona o entidad que contribuya con fondos, u otro tipo de apoyo material, al desarrollo del proceso en interés de la demanda o defensa

como sujetos activos o pasivos de una determinada pretensión, con prescindencia que revistan o no el carácter de sujetos legitimados para obrar o para contradecir en el concreto proceso que se trate. Al respecto, véase Lino Palacio, Derecho Procesal Civil, tom. 3 (Buenos Aires: Abeledo Perrot), 9-10.

(13) "International regulations are currently silent in connection with TPF, mainly because the funders are not comprised in any of the roles outlined under the arbitration rules and international conventions. Strictly speaking they are only investors, not subject to the responsibilities applicable to the key players involved in an international arbitration. Some commentators have enthusiastically argued that the parties would have an affirmative responsibility to disclose the presence of a funder even if the rules do not contain an explicit provision, in order to comply with the duty to participate in the arbitration proceeding with good faith". Sobre el particular, véase Francisco Blavi, "It's about time to regulate third party funding," Kluwer Arbitration Blog, 2015. (http://kluwerarbitrationblog.com/2015/12/17/its-about-time-to-regulate-third-party-funding/) 


\section{¿Tres son multitud? Algunas notas sobre el Third Party Funding y su aplicación en arbitrajes comerciales Three is a crowd? Some notes about Third Party Funding and its application in commercial arbitrations}

del caso, y que tenga un interés económico directo en, o un deber de indemnizar a una parte por, el laudo que se vaya a emitir en el arbitraje" (Directrices IBA sobre Conflictos de Intereses en Arbitraje Internacional 2014).

Se advirtió asimismo que:

"el deber de las partes de revelar cualquier relación directa o indirecta entre el árbitro y la parte (o cualquier otra sociedad del mismo grupo de compañías sobre un individuo con relación de control sobre la parte en el arbitraje) se ha ampliado a relaciones con personas o entidades con un interés económico directo en el laudo que será emitido en el arbitraje, tales como una entidad que financie el arbitraje o que tengan un deber de indemnizar a una parte por el laudo" (Directrices IBA sobre Conflictos de Intereses en Arbitraje Internacional 2014).

En consecuencia, las Directrices de la IBA del año 2014 generaron dos reglas claras de actuación: (i) el deber de revelar cualquier relación de los árbitros y las partes con financistas; y, (ii) la consecuencia jurídica de la actuación del tercero financista, para quien la norma que debe considerarse 'como si ostentara la identidad de la parte'.

A este esfuerzo inicial se han sumado otros intentos (aislados) de regular la figura, como el efectuado por la ICC en la Nota emitida el 12 de febrero de $2016^{(14)}$. Como bien indica la profesora Catherine Rogers, "hoy en día tenemos múltiples fuentes - incluyendo la Guía de la IBA, las Notas Prácticas de la ICC, las propuestas regulatorias en las leyes de Hong Kong y Singapur y algunos nuevos BIT's - que establecen estándares para revelar el financiamiento de terceros y de ese modo detectar posibles conflictos de intereses ${ }^{(15) "}$."

Sin embargo, debemos advertir que tanto las Notas Prácticas de la ICC como la Guía de la IBA son soft law y, en consecuencia, requieren ser recogidas por los Reglamentos Arbitrales de diversos Centros Arbitrales o incorporadas a las reglas del proceso.

Sumado a ello, no se puede extraer de esta normativa un deber general de revelar la existencia de un tercero financista, sino únicamente una obligación de relevación condicionada a la presencia de una relación previa entre la entidad financiadora y alguno de los árbitros, por lo que su alcance es, en el fondo, bastante acotado.

Algunos ordenamientos jurídicos (como los de Estados Unidos, Singapur y Hong Kong) ya han empezado a revisar la figura del third party funding (Jhangiani y Coldwell 2016). En la mayoría de casos se le ha sometido a múltiples restricciones, tales como: (i) la prohibición al inversionista de tomar control de los casos; (ii) prohibición de celebrar contratos entre el inversionista y los abogados patrocinantes si tales contratos no incluyen al cliente, (iii) prohibición a las firmas de abogados de financiar litigios; (iv) restricciones del uso de financiamiento de terceros para las demandas colectivas o class actions, entre otras ${ }^{(16)}$.

Resta señalar que ya en algunos casos de arbitrajes de inversiones se han emitido decisiones arbitrales destacando la relevancia e importancia de relevar la fuente del financiamiento asociado a reclamos arbitrales (Guaracachi America, Inc. and Rurelec PLC vs. Bolivia, UNCITRAL, PCA Caso No. 201117, Orden Procesal No. 13 del 21 de febrero

(14) Véase "ICC Courts Adopts Guidance Note on conflict disclosure by arbitrators". Nota de prensa disponible en iccwbo.org.

(15) "Today we have several new sources-including the IBA Guidelines, the ICC Practice Note, proposed Hong Kong and Singapore legislation, and some new BITs—which establish standards for disclosure of funders in order to assess potential arbitrator conflicts". Sobre el particular, véase Catherine Rogers, The World is Not Enough. En Kluwer Arbitration (2016): 2 y siguientes.

(16) Para un mayor detalle de estas restricciones y su aplicación en otros ordenamientos, véase John Beisnery Gary Rubin, "Stopping the Sale of Lawsuits: A proposal to regulate third party funding," Chamber Institute for Legal Reform (2012): 12 y siguientes. 
Carlos Ríos Pizarro

de 2013(17); Muhammet Çap \& Sehil Inşaat Endustri Ticaret Ltd. Sti. vs. Turkmenistan, Caso CIADI No. ARB/12/6, Orden Procesal No. 3 del 12 de junio de 2015; South American Silver vs. Bolivia, Caso PCA No. 2013-15, Orden Procesal No. 10 del 11 de enero de 2016).

La tendencia entonces parecer ser clara: a mayor información, mayor seguridad para las partes y menores restricciones y temores sobre el financiamiento de terceros. La solución, en nuestra opinión, debe apuntar a dicho norte.

\section{El presunto (e inexistente) impacto del Third Party Funding sobre las solicitudes de caución y de condena en costas}

En opinión de algunos juristas,"el Third Party Funding podría presentar un impacto real e importante en algunas actuaciones arbitrales clave, tales como las solicitudes de caución y de condena en costas" (Fernández 2016).

La solicitud de caución para costos (security for costs) es una figura utilizada en el common law que ha venido incorporándose poco a poco en el arbitraje internacional. Esta fianza es una medida cautelar que consiste en que la parte demandada exige al Tribunal Arbitral que la parte demandante pague una cantidad de dinero en concepto de fianzas con el fin de garantizar al solicitante el poder recuperar las costas del arbitraje si al final del arbitraje las pretensiones del demandante son rechazadas, siendo además esta parte condenada a pagar las costas de la otra.

Explica el profesor Fernández que:

"Los Tribunales Arbitrales, de forma tradicional y especialmente en controversias entre inversionistas y el Estado, se han mostrado desde siempre bastante reacios a conceder este tipo de solicitudes cuando así se les ha requerido. Sin embargo, la novedad que supone la presencia de un acuerdo de financiación de terceros podría ser un factor en el cambio de esta postura. Así, ha habido alguna opinión doctrinal que defiende que la existencia del Third Party Funding podría justificar que un tribunal arbitral ordenase que la parte contendiente preste caución suficiente para cubrir las costas derivadas del arbitraje" (Fernández 2016).

La lógica detrás de ello suele ser simple: si no hay dinero para cubrir los propios gastos, mucho menos habrá para reembolsar aquellos en los que ha incurrido la contraparte.

Por su parte, Born comenta que "la falta de recursos económicos de una parte para satisfacer una condena en costas, junto con que exista un acuerdo de financiación por terceros, constituye 'a strong prima facie case for security for costs” (Born 2014).

Sin embargo, en nuestra opinión la experiencia arbitral demuestra que la concesión de este tipo de medidas sigue siendo muy rara y excepcional, incluso con la aparición del Third Party Funding ${ }^{(18)}$.

Similar lógica se aplica para la condena en costos y costas. No existe fundamento, en nuestra opinión, para verse obligado a condenar a una parte en costos por el mero hecho de existir un acuerdo de financiamiento. La condena en costos dependerá de una serie de factores como el comportamiento de las partes durante el arbitraje, las reglas

(17) En octubre de 2015, Bolivia había solicitado al Tribunal Arbitral que ordenase a la parte demandante que revelase la identidad del financiador de este arbitraje, así como los términos del acuerdo de financiación. El Tribunal Arbitral aceptó la solicitud de Bolivia de la obligación de revelar el nombre de tercero financiador con el fin de otorgar transparencia al proceso. Sin embargo, rechazó obligar a la parte demandante a revelar los términos de su acuerdo de financiación. Al respecto, véase Caso CPA No. 2013-15, disponible en http://www.italaw.com/sites/default/files/case-documents/italaw7176.pdf.

(18) Sobre el particular, véase Stavros Brekoulakis, The Impact of Third Party Funding on Allocation for Costs and Securities for Costs Aplications. The ICCA- Queen Mary Task Force Report. Kluwer Arbitration Blog. 
¿Tres son multitud? Algunas notas sobre el Third Party Funding y su aplicación en arbitrajes comerciales Three is a crowd? Some notes about Third Party Funding and its application in commercial arbitrations

procesales aplicables, la estimación de los árbitros e incluso el propio pacto de las partes. En suma, no identificamos que dicha situación sea un problema que requiera de especial atención; a diferencia de los graves problemas que pudieran generarse derivados de los conflictos de intereses entre árbitros, partes $y$ terceros financistas.

\section{Conclusiones}

El financiamiento por terceros viene irrumpiendo con fuerza en el arbitraje internacional, y no nos cabe duda que pronto lo hará también en sede local. Los beneficios, como ya advertimos, son variados y podrían llegar a solucionar una parte del problema de acceso a la justicia en nuestro país.

Sin embargo, no pueden dejarse de lado los problemas que esta figura trae en lo que a conflictos de intereses respecta. La falta de una regulación precisa y específica que contemple las pautas a seguir en estos casos no hace sino acrecentar el problema. Tan es así que el $71 \%$ de los principales operadores jurídicos a nivel del arbitraje internacional cree que esta figura requiere una regulación urgente, como lo reveló la encuesta Queen Mary-White \& Case International Arbitration Survey del año 2015.

La comunidad arbitral ha reaccionado, pues, con temor sobre el uso de este esquema de financiamiento. Algunas críticas han sido muy válidas y otras, en nuestra opinión, bastante sencillas de superar. Empero, no puede ignorarse el hecho que el poder del financiamiento requiere también de mayor responsabilidad por parte de los diversos operadores jurídicos.
Felizmente, en los últimos años se ha iniciado una saludable tendencia para ampliar el deber de revelación de las partes y, de ese modo, incorporar el deber de revelar si se está siendo financiado por un tercero. Sacar a la luz aquellos conflictos, reales o potenciales, que pudieran afectar a cualquiera de las partes. Este, creemos, es el mejor remedio posible ante cualquier tipo de problema que se presente durante el arbitraje. Después de todo, y como alguna vez señaló Brandeis, la luz del sol es el mejor de los desinfectantes.

\section{Referencias bibliográficas}

Burke Robertson, Cassandra. 2011. The impact of Third Party Funding on Transnational Litigation. Case W. Reserve Journal of International Law. Vol 44: https://doi.org/10.2139/ ssrn.1966202

Born, Gary. 2014. International Commercial Arbitration. Wolter Kluwer.

Cremades, Bernando. 2011. Third Party Funding: Investing in Arbitration. ICC Institute of World Business Law 4, vol. 8.

Fernández Masiá, Enrique. 2016. La financiación por terceros en el arbitraje internacional. Cuadernos de Trabajo de la Universidad Carlos III de Madrid, 207- 217. https://doi. org/10.20318/cdt.2016.3256

Jhangiani, Sapna y Rupert Coldwell. 2016. Third-Party Funding for International Arbitration in Singapore and Hong Kong - A Race to the Top? Kluwer Arbitration Blog. http://kluwerarbitrationblog. com/2016/11/30/third-party-funding-for-international-arbitrationin-singapore-and-hong-kong-a-race-to-the-top/

Shanon Sahani, Victoria. 2016. Judging Third Party Funding. UCLA Law Review 63:391 -396. https://doi.org/10.2139/ ssrn. 2478245

Tripp Chapman y Daniel Kalderimis. 2014. Third party funding in international arbitration. Lessons from litigation? Kluwer Arbitration Blog. 